\title{
Characterizing Task-Related Temporal Dynamics of Spatial Activation Distributions in fMRI BOLD Signals
}

\author{
Bernard $\mathrm{Ng}^{1}$, Rafeef Abugharbieh ${ }^{1}$, Samantha J. Palmer ${ }^{2,3}$, \\ and Martin J. McKeown ${ }^{3}$ \\ ${ }^{1}$ Department of Electrical and Computer Engineering \\ ${ }^{2}$ Department of Neuroscience \\ ${ }^{3}$ Department of Medicine (Neurology), Pacific Parkinson's Research Center, \\ University of British Columbia, Vancouver, BC, Canada \\ bernardn@ece.ubc.ca, rafeef@ece.ubc.ca, \\ sjpalmer@interchange.ubc.ca, mmckeown@interchange.ubc.ca
}

\begin{abstract}
We present a new functional magnetic resonance imaging (fMRI) analysis method that incorporates both spatial and temporal dynamics of bloodoxygen-level dependent (BOLD) signals within a region of interest (ROI). 3D moment descriptors are used to characterize the spatial changes in BOLD signals over time. The method is tested on fMRI data collected from eight healthy subjects performing a bulb-squeezing motor task with their right-hand at various frequencies. Multiple brain regions including the left cerebellum, both primary motor cortices (M1), both supplementary motor areas (SMA), left prefrontal cortex (PFC), and left anterior cingulate cortex (ACC) demonstrate significant task-related changes. Furthermore, our method is able to discriminate differences in activation patterns at the various task frequencies, whereas using a traditional intensity based method, no significant activation difference is detected. This suggests that temporal dynamics of the spatial distribution of BOLD signal provide additional information regarding taskrelated activation thus complementing conventional intensity-based approaches.
\end{abstract}

Keywords: functional imaging, spatio-temporal fMRI analysis, region of interest (ROI), brain activation, 3D moments.

\section{Introduction}

The most common application of functional magnetic resonance imaging (fMRI) is in mapping neural region(s) to particular function(s) by examining which brain areas activate when a certain task is performed. Most conventional analysis methods, such as statistical parametric mapping (SPM) [1], analyze each voxel's timecourse independently and assign a statistics value to that voxel based on its probability of being activated. To make group inferences under this approach, spatial warping of each subject's brain to a common exemplar shape is often performed to create a correspondence between voxels across subjects [2]. However, spatial normalization, which is typically followed by spatial smoothing, may inappropriately pool responses 
from functionally dissimilar regions [3], thus degrading important spatial information. An alternative approach that involves drawing regions of interest (ROIs) individually for each subject, and examining the statistical properties of regional activation across subjects, has been shown to offer finer localization and increased sensitivity to task-related effects [3]. This subject-specific ROI-based approach is thus followed in this study.

To determine whether an ROI is activated or not, a simple approach is to calculate the average intensity over an ROI at every time point, and determine if the resulting average time course significantly correlates with the stimulus [4]. This approach, however, ignores any spatial information of activity within an ROI and assumes that only signal amplitude is modulated by task. However, spatial information might be an important attribute of brain activity. Preliminary evidence supporting this idea of spatial characterization was first shown by Thickbroom et al. [5], where the spatial extent of activation, as opposed to response magnitude, was found to be modulated by different levels of force during a sustained finger flexion task. Their results were based on visual inspection and counting the number of activated voxels within an ROI. Recently, we presented a more elaborate study of the spatial patterns of activity within an ROI where quantitative measures of invariant spatial properties were used to discriminate task-related differences in brain activity [6]. Results demonstrated that, by examining changes in different spatial aspects of an activation distribution, sensitivity in detecting functional changes is enhanced as compared to using intensity means only.

Previous analyses examining spatial patterns of activation, including that in [6], were performed on T-maps where the spatial information is collapsed over time, thus only considered the time-averaged spatial patterns of brain activity. In this paper, we extend our previously proposed spatial characterization approach to the temporal domain to explore whether the spatial distribution of the blood oxygenation leveldependent (BOLD) signal itself is modulated in time by task performance. We note an important difference between our current and previous work [6] is that the generated spatial feature time courses can be used to infer ROI activation, as opposed to only comparing 2 groups of time-averaged activation statistical maps. To characterize the spatial changes, three dimensional (3D) moment descriptors were used as features and were calculated at each time point. The magnitudes of these features, however, are normally not comparable across subjects due to inter-subject variability in brain shapes and sizes, but are comparable for the same subject over time. Thus, any detected modulations of the spatial features over time for a given subject may in fact represent meaningful spatial changes in activation.

In this study, eight healthy subjects were recruited to perform a bulb-squeezing task at various frequencies. The cerebellum, primary motor cortex (M1), supplementary motor area (SMA), prefrontal cortex (PFC), and anterior cingulate cortex (ACC) were chosen as regions of interest. We demonstrate that our method can both detect activation within an ROI, as well as discriminate differences in activation patterns at the various task frequencies. This confirms previous findings of the value in incorporating spatial information into traditional intensity-based fMRI analyses. 


\section{Data Acquisition and Preprocessing}

In this study, after informed consent was obtained, fMRI data were collected from 8 healthy subjects. Each subject was required to perform a right-handed motor task that involved squeezing a bulb with sufficient pressure such that an 'inflatable ring', shown as a black horizontal bar on a screen, was kept within an undulating pathway (Fig. 1-a). The pathway remains straight during rest periods and becomes sinusoidal at time of stimulus. Each run lasted $260 \mathrm{~s}$, consisting of a $20 \mathrm{~s}$ rest period at the beginning and end, 6 stimuli of $20 \mathrm{~s}$ duration, and $20 \mathrm{~s}$ rest periods between the stimuli, as shown in Fig. 1-b. At time of stimulus, the subject was required to squeeze the bulb at $0.25,0.5$ or $0.75 \mathrm{~Hz}$, corresponding to 'Slow', 'Med', and 'Fast' in Fig 1b. The data were collected as part of a larger experiment exploring the rate of change of force production in older subjects and subjects with Parkinson's disease.

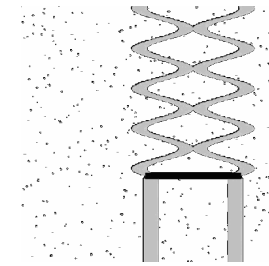

(a)

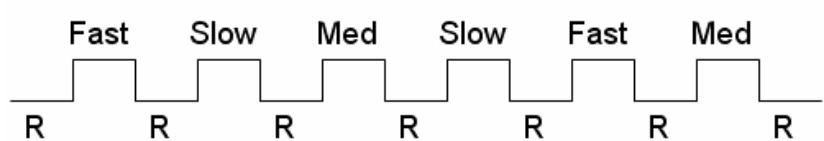

(b)

Fig. 1. Experimental task and stimulus timing. (a) Subjects were required to keep the side of the black ring on the gray path (see text). (b) $\mathrm{R}=$ rest, Slow, Med, and Fast = stimulus at $0.25,0.5$, and $0.75 \mathrm{~Hz}$, respectively. Each block is $20 \mathrm{~s}$ in duration.

\section{1 fMRI Data Acquisition}

Functional MRI was performed on a Philips Gyroscan Intera 3.0 T scanner (Philips, Best, Netherlands) equipped with a head-coil. We collected echo-planar (EPI) T2*weighted images with BOLD contrast. Scanning parameters were: repetition time $1985 \mathrm{~ms}$, echo time $3.7 \mathrm{~ms}$, flip angle $90^{\circ}$, field of view (FOV) $216 \times 143 \times 240 \mathrm{~mm}$, in plane resolution $128 \times 128$ pixels, pixel size $1.9 \times 1.9 \mathrm{~mm}$. Each functional run lasted 4 minutes where 36 axial slices of $3 \mathrm{~mm}$ thickness were collected in each volume, with a gap thickness of $1 \mathrm{~mm}$. We selected slices to cover the dorsal surface of the brain and included the cerebellum ventrally. A high resolution 3D T1-weighted image consisting of 170 axial slices was acquired of the whole brain to facilitate anatomical localization of activation for each subject.

\section{2 fMRI PreProcessing}

The fMRI data was preprocessed for each subject, using Brain Voyager's (Brain Innovation B.V.) trilinear interpolation for 3D motion correction and sinc interpolation for slice time correction. Further motion correction was performed using motion corrected independent component analysis (MCICA) [7]. To handle temporal autocorrelations, a 'coloring' scheme was used [8], where the time series were high-pass 
filtered at $0.02 \mathrm{~Hz}$ (task-frequency being $0.025 \mathrm{~Hz}$ ) to remove the majority of the low frequency noise, and temporally smoothened with a Gaussian of width $2.8 \mathrm{~s}$ [8]. The first and last $20 \mathrm{~s}$ of the time series were truncated to mitigate transient effects. No spatial smoothing was performed.

The Brain Extraction Tool (BET) in MRIcro [9] was used to strip the skull off of the anatomical and first functional image from each run to enable a more accurate alignment of the functional and anatomical scans. Custom scripts to co-register the anatomical and functional images were generated using the Amira software (Mercury Computer Systems, San Diego, USA).

Ten specific ROIs were manually drawn on each unwarped structural scan using Amira. The following ROIs were drawn separately in each hemisphere, based upon anatomical landmarks and guided by a neurological atlas [10]: cerebellum, M1 (Brodman Area 4), SMA (Brodman Area 6), PFC (Brodman Area 9 and 10), and ACC (Brodman Area 28 and 32). The labels on the segmented anatomical scans were resliced at the fMRI resolution. The raw time courses of the voxels within each ROI were then extracted for analysis as described in the next section.

\section{Methods}

The main goal of the proposed method is to demonstrate that temporal dynamics of the spatial distribution in BOLD signals can be used to infer whether an ROI is activated, as well as to discriminate differences in activation patterns at various task frequencies. Details of feature time course extraction, activation detection, and activation pattern discrimination are discussed below.

\subsection{Feature Time Course Extraction}

The spatial feature descriptors used in this paper are based on centralized 3D moments, defined as:

$$
\mu_{p q r}(t)=\int_{-\infty}^{\infty} \int_{-\infty}^{\infty} \int_{-\infty}^{\infty}(x-\bar{x})^{p}(y-\bar{y})^{q}(z-\bar{z})^{r} \rho(x, y, z, t) d x d y d z
$$

where $n=p+q+r$ is the order of the moment, $(x, y, z)$ are the coordinates of a voxel, $\rho(x, y, z, t)$ is the intensity of a voxel located at $(x, y, z)$ inside a given ROI at time $t$, and $\bar{x}, \bar{y}$, and $\bar{z}$ are the centroid coordinates of $\rho(x, y, z, t)$. To untangle the effect of amplitude changes, $\rho(x, y, z, t)$ is normalized such that the intensity values of the voxels within the ROI sums up to one at every time point $t$. This step ensures that the mean ROI intensity does not change with time. Thus, any detected modulations in the spatial feature will be purely due to spatial changes in the BOLD signal. To ease interpretation of the results and since higher order moments are less robust to noise [11], only $2^{\text {nd }}$ and $3^{\text {rd }}$ order $3 \mathrm{D}$ moment descriptors characterizing spatial variance [12] and skewness, respectively, were used:

$$
J_{1}(t)=\mu_{200}(t)+\mu_{020}(t)+\mu_{002}(t)
$$




$$
S(t)=\mu_{300}(t)+\mu_{030}(t)+\mu_{003}(t),
$$

To compare with the results obtained using the proposed spatial feature time courses, the traditionally used mean intensity time course, $I(t)$, for each ROI of a given subject is calculated by averaging the intensity over the ROI at every time point.

\subsection{Activation Detection}

To make group inference as to whether a given ROI is activated, each subject's ROI feature time courses (spatial or mean intensity) are first correlated with a box-car that is time-locked to stimulus with a delay of $4 \mathrm{~s}$ [13]. We did not convolve the box-car with a haemodynamic response function since spatial changes, as governed by the different onsets of the active voxels, may exhibit a different temporal profile than that of the haemodynamic response. For each subject, this results in thirty correlation values, one per combination of feature and ROI (e.g. $J_{l}(t)$, left M1). Each correlation value is then converted into a T-value (4):

$$
T=|r| \sqrt{\frac{N-2}{1-r^{2}}},
$$

where $r$ is the correlation value and $N$ is the number of samples used in generating $r$. The set of T-values of a particular combination of feature and ROI from all subjects is then tested against 1.96 using a T-test to determine the probability (p-value) that the $\mathrm{T}$-values are lower than 1.96 (i.e. the probability that ROI is not activated). The critical p-value was chosen at 0.05 .

\subsection{Activation Pattern Discrimination}

To discriminate the differences in activation pattern at the various task frequencies, each subject's ROI feature time courses (spatial or mean intensity) are first segmented according to Fig. 2. Except for the first and last segments, each segment consists of a

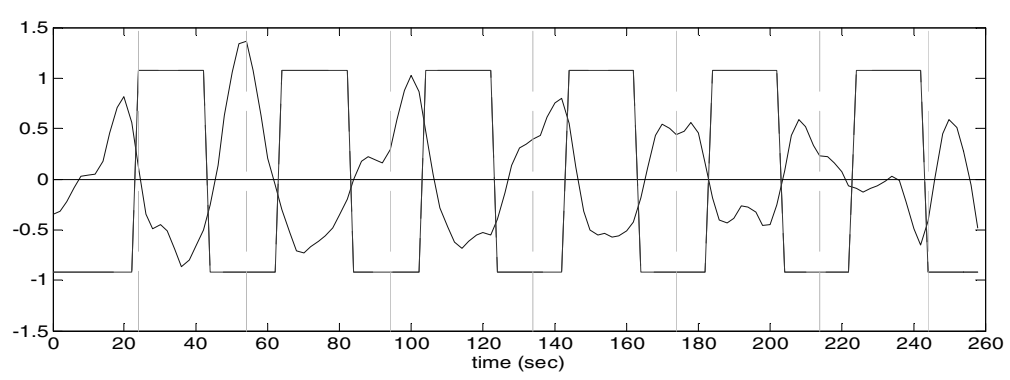

Fig. 2. Feature time course segmentation. The box-car curve corresponds to timing of the stimulus delayed by $4 \mathrm{~s}$. The solid line is a sample feature time course (spatial variance, $J_{l}(t)$, of the left M1 averaged over subjects with its temporal mean removed and divided by its standard deviation). The dotted lines show how the feature time courses are parsed into 6 segments. Slow, Med, and Fast correspond to the task frequencies of $0.25,0.5$, and $0.75 \mathrm{~Hz}$, respectively. 
$10 \mathrm{~s}$ rest before and after the $20 \mathrm{~s}$ stimulus. Segments of the same task frequency are concatenated and correlated with the corresponding segments of the shifted reference signal (see Fig. 2). This results in ninety correlation values per subject, one for each combination of frequency, feature, and ROI (e.g. slow, $J_{l}(t)$, left M1). Each correlation value is then converted into a T-value using (4).

For each combination of feature and ROI, the set of T-values of a particular frequency from all subjects are tested pair-wise against the other two frequencies (i.e. fast versus slow, fast versus medium, medium versus slow). This is performed using a T-test to determine the probability ( $\mathrm{p}$-value) that the sets of T-values from the two task frequencies are the same (i.e. the probability that activation patterns at the two frequencies are the same). The critical p-value was chosen at 0.05 .

\section{Results and Discussion}

Table 1 summarizes the activation detection results generated by extracting the spatial and intensity features from real fMRI data as described in Section 3.2, and correlating the resulting feature time courses with the reference signal.

Table 1. p-values of ROI activation. CER = cerebellum, $J_{l}(t)=$ spatial variance, $S(t)=$ skewness, $I(t)=$ mean intensity, $\mathrm{L}=$ left, $\mathrm{R}=$ right, $*$ = statically significant at $\alpha=0.05$

\begin{tabular}{cccc}
\hline Feature & $J_{1}(t)$ & $S(t)$ & $\bar{I}(t)$ \\
\hline LCER & $\mathbf{0 . 0 0 2} *$ & 0.077 & 0.469 \\
\hline RCER & 0.220 & 0.066 & $\mathbf{0 . 0 4 2}$ \\
\hline LM1 & $\mathbf{0 . 0 0 2}^{*}$ & 0.051 & 0.162 \\
\hline RM1 & 0.157 & $\mathbf{0 . 0 4 7}^{*}$ & 0.164 \\
\hline LSMA & $\mathbf{0 . 0 2 7}$ & $\mathbf{0 . 0 3 4}^{*}$ & $\mathbf{0 . 0 2 1}$ \\
\hline RSMA & $\mathbf{0 . 0 4 4}$ & $\mathbf{0 . 0 3 5 ^ { * }}$ & $\mathbf{0 . 0 3 4}$ \\
\hline LPFC & $\mathbf{0 . 0 3 6} *$ & $\mathbf{0 . 0 2 0}$ & $\mathbf{0 . 0 3 4}$ \\
\hline RPFC & 0.341 & 0.185 & 0.058 \\
\hline LACC & $\mathbf{0 . 0 0 1 *}$ & 0.301 & $\mathbf{0 . 0 2 3}$ \\
\hline RACC & 0.056 & 0.112 & $\mathbf{0 . 0 4 5}$ \\
\hline
\end{tabular}

Using spatial variance, $J_{l}(t)$, the left cerebellum, left M1, both SMAs, left PFC, and left ACC were detected as active. We expected the left M1 to be activated, as typically observed for right-handed motor tasks. It is worth noting that the left M1's BOLD signal distribution shown reduced spatial variance (i.e. focuses) during the time of stimulus (see Fig. 2). Skewness, $S(t)$, additionally detected activation in the right M1. These results demonstrate that the spatial distribution of BOLD signals is, in fact, modulated by stimulus, which supports our hypothesis that spatial changes in BOLD signals are task-related and can be used to infer activation within an ROI.

Using the traditional mean intensity measure, the right cerebellum, both SMAs, left PFC, and both ACCs were detected as active. Comparing to the results generated with the proposed spatial features, some consistencies are shown. In fact, based on the results in Table 1, activation within an ROI appears to modulate both in amplitude and in space. 
Segmenting the feature time courses according to task frequencies and using the proposed spatial features, significant frequency-related activation differences were detected in the right cerebellum and right M1 when comparing fast versus slow frequencies (Table 2). These results matched our expectations since the modulation of movement speed is known to involve a complex network of brain areas, including the right cerebellum and right M1 [14]. Also, significant activation differences were found in the right PFC and right ACC using the proposed spatial features. In contrast, no significant activation differences were found using mean intensity. Also, no significant activation differences were detected when comparing fast versus medium frequencies and medium versus slow frequencies for any of the features, thus these results were excluded in Table 2.

Table 2. p-values of activation differences comparing fast versus slow frequencies. CER = cerebellum, $J_{l}(t)=$ spatial variance, $S(t)=$ skewness, $\vec{I}(t)=$ mean intensity, $\mathrm{L}=$ left, $\mathrm{R}=$ right, *statically significant at $\alpha=0.05$.

\begin{tabular}{cccc}
\hline Feature & $J_{1}(t)$ & $S(t)$ & $\bar{I}(t)$ \\
\hline LCER & 0.1834 & 0.1931 & 0.1528 \\
\hline RCER & 0.9993 & $\mathbf{0 . 0 0 9 6}$ & 0.2757 \\
\hline LM1 & 0.2932 & 0.4017 & 0.4190 \\
\hline RM1 & $\mathbf{0 . 0 4 4 2 *}$ & 0.3223 & 0.1524 \\
\hline LSMA & 0.6872 & 0.2499 & 0.1836 \\
\hline RSMA & 0.7887 & 0.9066 & 0.0579 \\
\hline LPFC & 0.5981 & 0.3059 & 0.2760 \\
\hline RPFC & $\mathbf{0 . 0 3 9 8 *}$ & 0.3668 & 0.7416 \\
\hline LACC & 0.2551 & 0.4029 & 0.2126 \\
\hline RACC & $\mathbf{0 . 0 3 1 1 ^ { * }}$ & 0.1028 & 0.3248 \\
\hline
\end{tabular}

Examining the results in Table 2, spatial changes appear to provide greater sensitivity in detecting subtle activation differences as compared to intensity.

\section{Conclusions}

In this paper, we proposed using 3D moment-based spatial descriptors to characterize the temporal dynamics of spatial activation distribution within an ROI for fMRI analysis. We demonstrated with real fMRI data that certain spatial aspects of activation, as opposed to just amplitude, are modulated by stimulus - a result that appeared consistent across subjects. Furthermore, we showed that our method was able to better discriminate frequency-related differences in activation patterns during motor task performance when compared to using mean intensity only. These results suggest that spatial characterization of BOLD signal can complement traditional intensity-based fMRI analysis. A direct extension of the proposed method would be to examine functional connectivity and phase relations between ROIs using spatial feature time courses, an approach currently being pursued. 


\section{References}

1. Friston, K.J., Toga, A.W., Mazziotta, J.C. (eds.): Statistical parametric mapping and other analyses of functional imaging data. Brain mapping, the methods, pp. 363-396. Academic Press, San Diego (1996)

2. Lancaster, J.L., Rainey, L.H., Summerlin, J.L., Freitas, C.S., Fox, P.T., Evans, A.C., Toga, A.W., Mazziotta, J.C.: Automated labeling of the human brain: a preliminary report on the development and evaluation of a forward-transform method. Hum. Brain Mapp. 5(4), 238 242 (1997)

3. Castanon, A.N., Ghosh, S.S., Tourville, J.A., Guenther, F.H.: Region of interest based analysis of functional imaging data. NeuroImage 19, 1303-1316 (2003)

4. Liu, Y., Gao, J.H., Liotti, M., Pu, Y., Fox, P.T.: Temporal dissociation of parallel processing in the human subcortical outputs. Nature 400(6742), 364-367 (1999)

5. Thickbroom, G.W., Phillips, B.A., Morris, I., Byrnes, M.L., Mastaglia, F.L.: Isometric force-related activity in sensorimotor cortex measured with functional MRI. Exp. Brain Res. 121, 59-64 (1998)

6. Ng, B., Abugharbieh, R., Huang, X., McKeown, M.J.: Characterizing fMRI Activations within Regions of Interest (ROIs) Using 3D Moment Invariants. In: IEEE Worshop on Mathematical Methods in Biomedical Image Analysis, New York (June 17-18, 2006)

7. Liao, R., Krolik, J.L., McKeown, M.J.: An information-theoretic criterion for intrasubject alignment of fMRI time series: Motion corrected independent component analysis. IEEE Trans. Med. Imaging 24, 29-44 (2005)

8. Friston, K.J., Holmes, A.P., Poline, J.B., Grasby, P.J., Williams, S.C.R., Frackowiak, R.S.J., Turner, R.: Analysis of fMRI Time-Series Revisited. NeuroImage 2, 45-53 (1995)

9. Rorden, C., Brett, M.: Stereotaxic display of brain lesions. Behavioural Neurology 12, 191-200 (2000)

10. Talairach, J., Tournoux, P.: Co-Planar Stereotaxic Atlas of the Human Brain: 3Dimensional Proportional System - an Approach to Cerebral Imaging. Thieme Medical Publishers, New York (1988)

11. Press, W.H., Teukolsky, S.A., Vetterling, W.T., Flannery, B.P.: Nurmerical Recipes in C. Cambridge University Press, Cambridge (1999)

12. Sadjadi, F.A., Hall, E.L.: Three-Dimensional Moment Invariants. IEEE Trans. Pattern. Anal. Machine Intell. PAMI- 2, 127-136 (1980)

13. Svensen, M., Kruggel, F., von Cramon, Y.: Probabilitic modeling of single-trial fMRI data. IEEE Trans. Med. Imaging 19(1), 25-35 (2000)

14. Verstynen, T., Diedrichsen, J., Albert, N., Aparicio, P., Ivry, R.B.: Ipsilateral motor cortex activity during unimanual hand movements relates to task complexity. J. Neurophysiol. 93, 1209-1222 (2005) 